\title{
ETUDE QUANTITATIVE DU BENTHOS D'UNE RIVIẼRE DE BELGIQUE : L'OURTHE LIÉGEOISE
}

\author{
par J.-C. MichA
}

SOMMAIRE

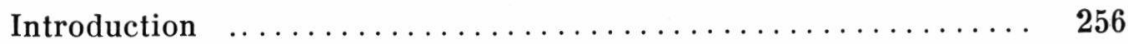

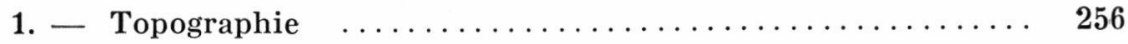

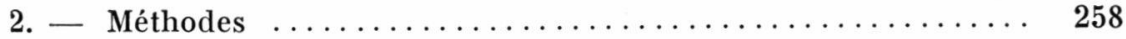

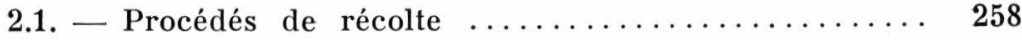

- Au niveau des rapides $\ldots \ldots \ldots \ldots \ldots \ldots \ldots \ldots .258$

- Au niveau des stations de barrage .......... 259

2.2. - Traitement du matériel récolté ............. 259

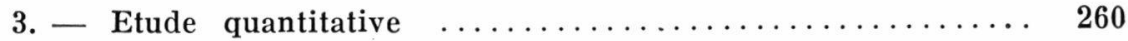

3.1. - Structure biocénotique $\ldots \ldots \ldots \ldots \ldots \ldots \ldots \ldots .260$

- Stations d'eau courante $\ldots \ldots \ldots \ldots \ldots \ldots \ldots \ldots .260$

- Stations de barrage $\ldots \ldots \ldots \ldots \ldots \ldots \ldots \ldots \ldots .266$

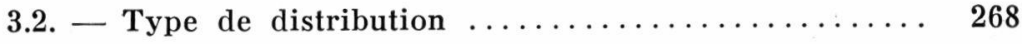

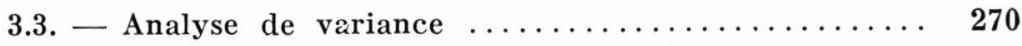

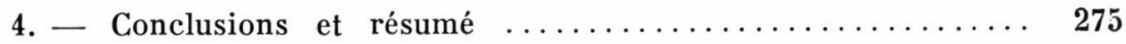




\section{INTRODUCTION}

Le présent article constitue le troisième volet d'une étude des populations d'Invertébrés de l'Ourthe liégeoise. Un premier travail a été consacré à l'exposé des caractères physico-chimiques de Ia rivière [MIcha, 1970]. Une seconde publication a été réservée à une analyse qualitative de la composition des communautés animales benthiques [Micha, 1969]. Cette troisième étude, qui se rapporte également à l'ensemble des Invertébrés du benthos de l'Ourthe, a pour objet de déterminer, à l'aide du calcul statistique, différents caractères écologiques tels que : structure biocénotique, biomasse comparée dans différents habitats et types de distribution de quelques espèces dans ces mêmes habitats. A ce propos, rappelons que la structure biocénotique donne une idée de l'abondance relalive des différentes espèces qui vivent dans un habitat déterminé, que la biomasse comparée permet de faire un parallèle entre les quantités de matière vivante existant à un moment donné en des endroits différents et enfin que le type de distribution nous éclaire sur la structure interne d'une population dans l'habitat qu'elle occupe [VIBERT et LAGLER, 1961]. Enfin, l'analyse de variance doit nous permettre de tester les différences observées entre les communautés dans différentes stations d'un même habitat.

Qu'il nous soit permis de remercier les dirigeants de la Commission provinciale de Liège du Fonds Piscicole pour la bourse de recherches qu'ils nous ont accordée. Il nous est agréable d'exprimer notre reconnaissance à M. le Professeur J.-C. Ruwer, qui nous a donné toutes les facilités de travail dans son laboratoire ainsi qu'à M. L. NEF, pour l'aide précieuse qu'il nous a apportée dans l'analyse statistique.

\section{1. - TOPOGRAPHIE DE LA RIVIERE}

L'Ourthe est une grosse rivic̀re ardennaise du bassin de la Meuse. Elle provient de la réunion de deux rivières de Haute Belgique, longues d'environ $50 \mathrm{~km}$ : l'Ourthe orientale et occidentale qui confluent à Engreux. Elle est alors considérée comme une grosse rivière, et parcourt $135 \mathrm{~km}$ avant de se jeter dans la Meuse à Liège, en passant de l'altitude $270 \mathrm{~m}$ (Engreux) à l'altitude $65 \mathrm{~m}$.

Dans ce travail, nous nous limitons à l'étude de l'Ourthe inférieure dans la Province de Liège (fig. 1) à savoir depuis Bomal $(2,2 \mathrm{~km}$ en aval) jusqu'à son embouchure dans la Meuse, soit un parcours de $47,5 \mathrm{~km}$. Sur ce trajet. l'Ourthe traverse différentes localités dont les plus importantes sont Hamoir, Comblain-au-Pont, Esneux, Tilff et Angleur. 


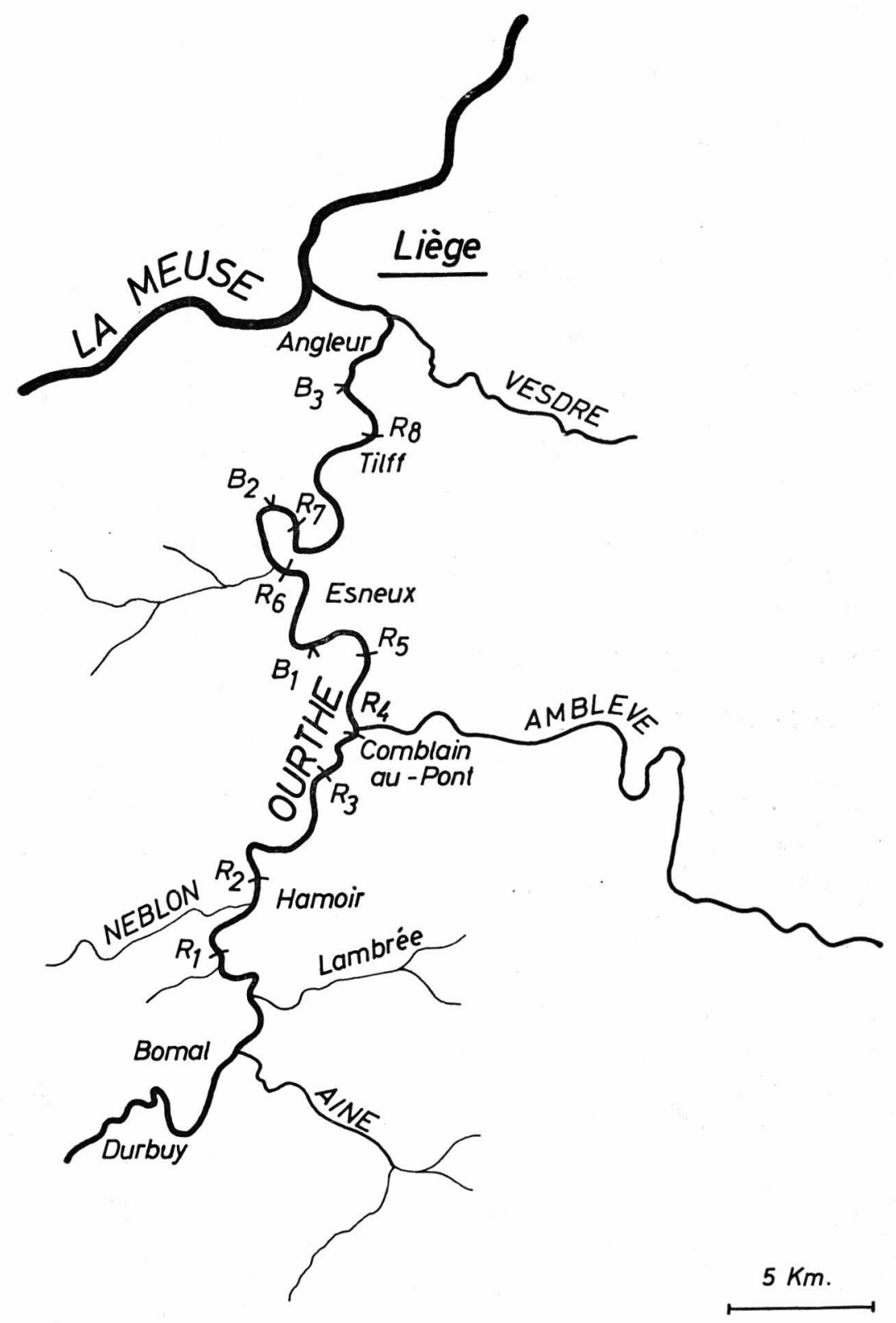

Topographie de l'Ourthe liégeoise. 
L'observation du parcours de l'Ourthe nous a amené à choisir deux types de sites représentatifs de cette rivière : les sites rapides (R), particulièrement nombreux sur la partie supérieure de son cours dans la province de Liège, et les sites calmes au niveau des petits barrages artificiels $(1,5-3 \mathrm{~m})$.

Parmi les 57 rapides repérés sur le parcours de l'Ourthe liégeoise, huit ont été choisis en fonction de leur richesse floristique et faunistique et de leur localisation par rapport aux localités les plus importantes susceptibles de la polluer.

Ces huit rapides sont groupés chaque fois en amont et en aval de Hamoir, Comblain-au-Pont, Esneux et Tilff (fig. 1).

Parmi les dix barrages ou déversoirs modifiant le cours de l'Ourthe, trois seulement ont été prospectés. Ce sont : le barrage de la Gombe $B_{1}$, le barrage d'Esneux $B_{2}$, et the barrage de Colonster $\mathbf{B}_{3}$ (fig. 1).

\section{2. - MÉTHODES D'ÉTUDE}

Les méthodes utilisées sont très simples et nécessitent un matériel assez restreint.

\section{1. - Procédés de récolte.}

Pour les récoltes, nous utilisons l'appareil classique de Surber [WeLch, 1952] de $25 \mathrm{~cm}$ de côté et pourvu du filet français $\mathrm{n}^{\circ} 30$, à mailles de $750 \mu$. La dimension des mailles est suffisamment petite pour retenir tous les organismes qui nous intéressent, car même les oligochètes de diamètre plus petit s'emmêlent dans les mailles du filet et peuvent faire l'objet d'un comptage valable.

\section{- RÉcoltes au niveau des RAPIdes (R)}

A chaque station, 8 échantillons sont prélevés, dont 4 sont effectués sur cailloux et gravier et 4 à partir des herbiers à Ranunculus fluitans LmK. Ceux-ci sont découpés selon un carré de $25 \mathrm{~cm}$ de côté au moyen d'une palette de plâtrier affutée. Le gravier et la vase sous-jacente sont repoussés à la main dans le filet. Chaque série des 4 prélèvements est effectuée au hasard sur un tronçon de rivière de $150 \mathrm{~m}$ environ de longueur.

Le matériel récolté est alors transvasé dans des bassins de plastique à fond quadrillé permettant ainsi un comptage assez précis. Nous avons essayé différents traitements chimiques et notamment l'emploi de $\mathrm{CaCl}_{2}$ qui, d'après la littérature [MaCAN, 1958], amène par différence de densité tous les organismes en surface. Mais ces procédés ne nous ont jamais donné satisfaction; aussi, avons-nous 
préféré effectuer les comptages par observation minutieuse soit du matériel frais, soit du matériel gardé au maximum deux jours en réfrigération à $5^{\circ} \mathrm{C}$.

\section{- Récoltes au niveau des stations de barrage (B).}

La présence des petits barrages, aussi appelés déversoirs, dont la hauteur varie de $1,50 \mathrm{~m}$ à $3 \mathrm{~m}$ environ, maintient en amont un plan d'eau plus ou moins considérable. Les macro-végétaux, tels que Ranunculus et Potamogeton, ne se développent jamais dans de telles conditions, aussi l'ensemble des 8 prélèvements sont toujours effectués sur cailloux, gravier et vase.

La profondeur, toujours importante, ne permet plus le prélèvement classique avec l'appareil de Surber. Aussi, avons-nous effectué les prélèvements en plongée avec l'appareil de Surber. Le processus est simple : il suffit de fixer et de tendre en travers de la rivière une longue corde sur laquelle coulisse un câble qui maintient le plongeur, face au courant. Celui-ci, muni de l'appareil de Surber, prélève à son aise les matériaux de fond, torsade le filet et mène le tout sur la berge où se fait le transvasement en bassin plastique. La suite des opérations a lieu alors comme cidessus.

\section{2. - Traitement du matériel récolté.}

Il nous a semblé inutile de mesurer le poids frais des Invertébrés aquatiques récoltés. En effet, le poids frais donne une trop vague idée de la quantité de matières organiques chez les Invertébrés aquatiques imbibés d'eau à souvent plus de $80 \%$. Aussi, dans un milieu où cet élément ne fait jamais défaut, il nous parait vain de mesurer le poids frais d'autant plus que celui-ci varie fortement selon que les individus sont plus ou moins égouttés ou séchés.

C'est pourquoi, afin de mieux cerner l'importance relative des différentes espèces et de tenter une meilleure approche de la biomasse "secondaire", nous avons recueilli de nombreux individus de toute taille pour en mesurer le poids sec.

Tous les organismes ont été tués dans l'alcool à $40^{\circ}$. Ils ont ensuite été lavés et nettoyés au pinceau puis conservés dans l'alcool à $60^{\circ}$. Ce produit conserve parfaitement les organismes et dissout tout au plus les pigments ce qui, du point de vue poids des individus, est insignifiant.

Les individus ainsi récoltés ont ensuite été placés dans des pèse-substances appropriés. Les mollusques ont évidemment été pesés avec leur coquille, les larves de trichoptères ont été débarrassées de leur fourreau sauf celles de Brachycentrus. En effet, 
ces larves délicates cassaient lors de l'extraction hors du fourreau et nous avons préféré les peser dans leur habitat végétal.

La déshydratation des organismes a été effectuée à $100^{\circ} \mathrm{C}$ dans une étuve Bekso de type E 55, de voltage égal à $110 \mathrm{~V}$ et de $1800 \mathrm{KW}$ de puissance. Cette déshydratation a duré jusqu'à stabilisation des pesées. Le poids a été mesuré au $1 / 10^{\mathrm{e}} \mathrm{de} \mathrm{mgr}$ au moyen d'une balance Sartorius Werke modèle J 1/200 g.

\section{3. - ETUDE QUANTIT.ATIVE.}

L'aspect quantitatif des populations de macroinvertébrés de l'Ourthe liégeoise est tout d'abord examiné au point de vue biocénotique et biomasse. Les communautés des sites de rapides amont et aval sont comparées deux à deux puis un parallèle est fait avec les communautés des stations de barrage. Ensuite, le type de distribution des espèces les plus abondantes est vérifié et l'analyse de variance est effectuée sur les données normalisées par transformation logarithmique.

\section{1. - Structure biocénotique et biomasse.}

La structure biocénotique des communautés d'Invertébrés a été examinée aux différentes stations d'eau courante et de barrage et le poids sec par taxons a été évalué en gramme $/ \mathrm{m}^{2}$.

\section{- Stations d'eau courante.}

Les divers sites d'eau courante, à savoir amont et aval. des 4 villes (Hamoir, Comblain-au-Pont, Esneux et Tilff) déversant leurs affluents dans l'Ourthe liégeoise supérieure, sont comparés deux à deux.

1. - Le tableau I, relatif aux stations $R_{1}$ et $R_{2}$ respectivement amont et aval de Hamoir, met en évidence un accroissement net des populations d'Invertébrés en aval de la ville. Cette augmentation du nombre d'individus se manifeste surtout chez l'Oligochète Lumbriculus variegatus, les Achètes et notamment Erpobdella octoculata, les Mollusques Ancylus fluviatilis, et Lymnea auricularia, le Trichoptère Hydropsyche $s p$. et les Diptères. Les autres groupes gardent plus ou moins leur statu quo.

L'examen de la biomasse montre également une augmentation nette. Les taxons en cause sont, dans l'ordre d'importance, les Mollusques Ancylus fluviatilis et Lymnea auricularia, l'Achète Erpobdella octoculata, le Trichoptère Hydropsyche sp., le Diptère Simulium equinum, etc... 
Dans l'ensemble, la structure biocénotique des deux stations reste fort semblable. En effet, dans chaque cas, les taxons les plus importants au point de vue densité sont dans l'ordre décroissant: Simulium equinum, Brachycentrus sp., Chironomus sp., Hydropsyche sp. et Lumbriculus variegatus.

2. - Comblain-au-Pont (tableau I) présente un nombre global d'individus plus élevé à sa station amont $R_{3}$ qu'à sa station aval $R_{4}$. Cette légère diminution en aval se manifeste notamment chez l'Oligochète Lumbriculus variegatus, les Ephémères nageuses et les Diptères dont Simulium equinum et Chironomus spp. Par contre, différents taxons contrebalancent cette perte par une densité de population plus grande : Ancylus fluviatilis, Theodoxus fluviatilis, Gammarus pulex, Brachycentrus sp., etc. La plupart des autres taxons montrent approximativement la même densité de population.

$\mathrm{Si}$ la densité tolale des individus diminue à la station amont, paradoxalement la biomasse augmente légèrement. Ceci provient du fait que la diminution de densité de population à la station aval se manifeste surtout pour des taxons dont le poids sec par individu est très faible. Par contre, l'augmentation relativement faible de la densité des Mollusques permet un accroissement considérable de la biomasse. C'est pourquoi il ne faut pas s'étonner que le bilan d'ensemble mette en évidence une biomasse plus élevée à la station aval malgré une densité totale d'individus plus faible.

Quant à la structure biocénotique des deux stations, elle n'apparaît pas aussi stable qu'à Hamoir. Toutefois, les taxons les plus importants au point de vue densité restent approximativement les mêmes dans les deux stations.

3. - L'examen du tableau II montre, en aval d'Esneux, un accroissement net de la densité totale des populations d'Invertébrés. Toutefois, cette différence importante provient surtout du recrutement en jeunes individus de Brachycentrus sp. Les autres taxons qui présentent une augmentation importante de densité de population en aval d'Esneux sont : les Ephémères nageuses, Hydropsyche sp. et Simulium equinum. Notons cependant que la densité de population de quelques taxons diminue : c'est le cas de Lumbriculus variegatus, Sphaerium corneum et Chironomus sp. De toute façon, le bilan général montre un accroissement du nombre d'individus même si l'on défalque les individus de Brachycentrus sp.

La biomasse augmente également en aval d'Esneux. Pour la première fois, cet accroissement ne se manifeste pas chez les Mollusques mais provient surtout du groupe des Ephémères fouis- 


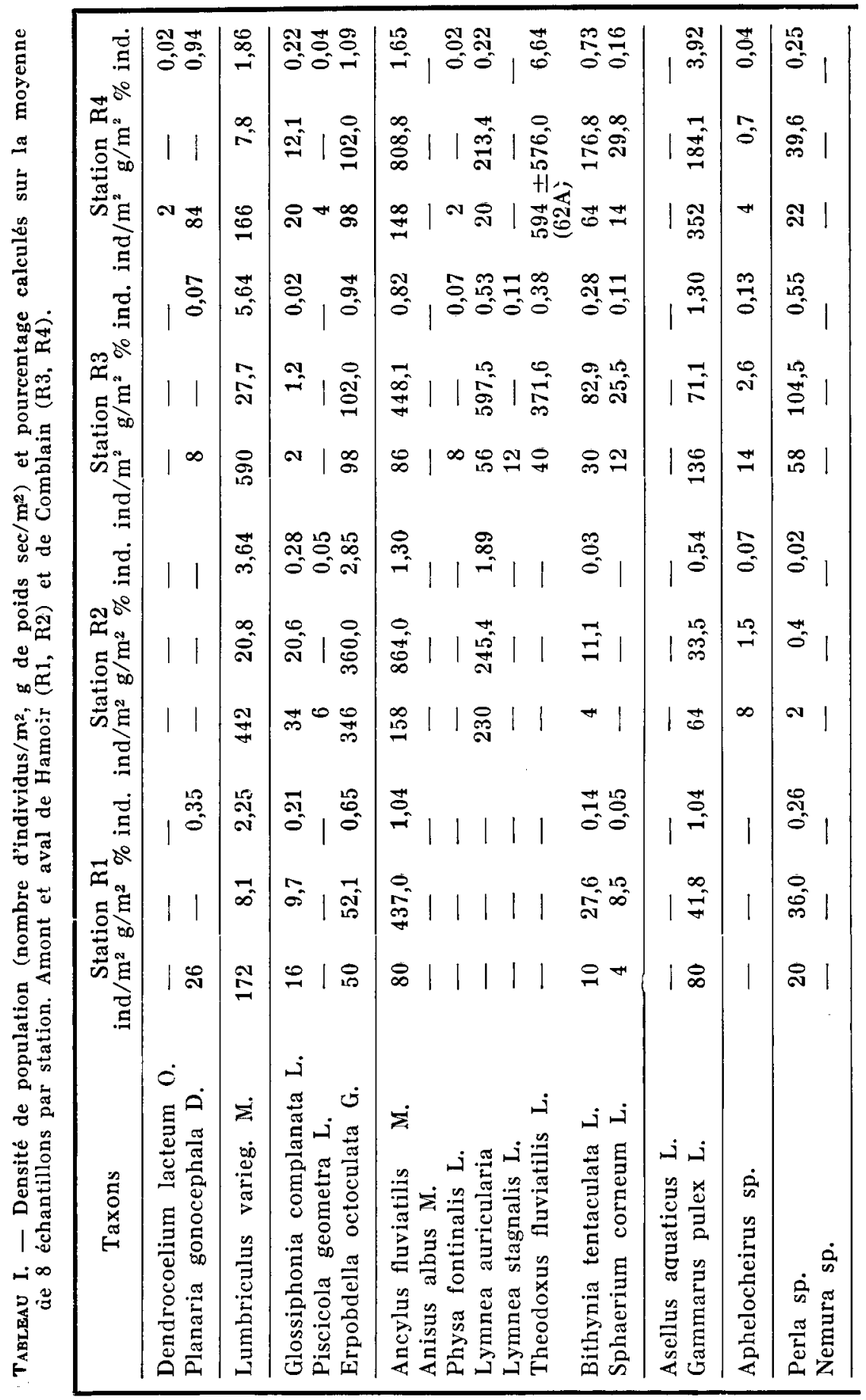




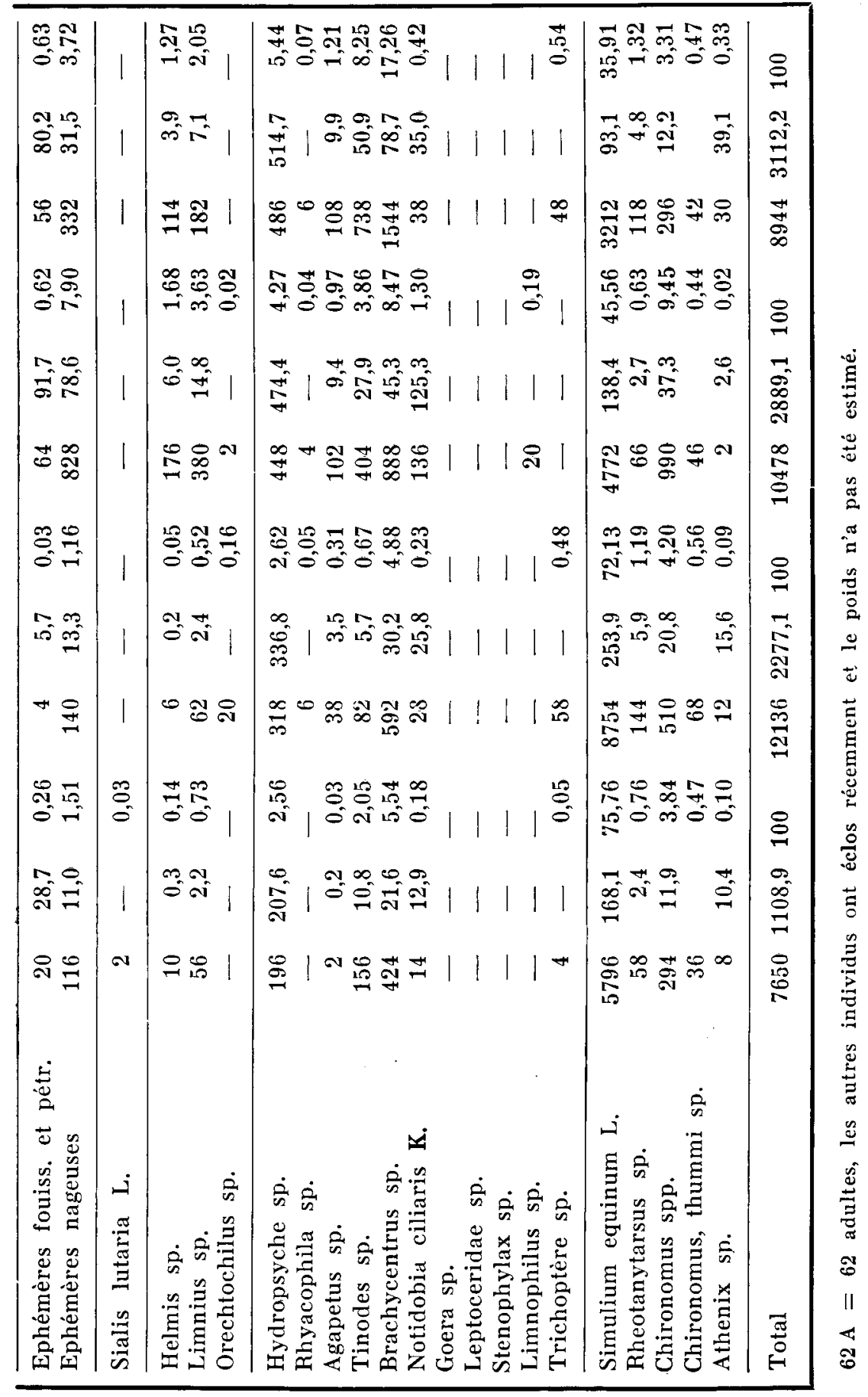




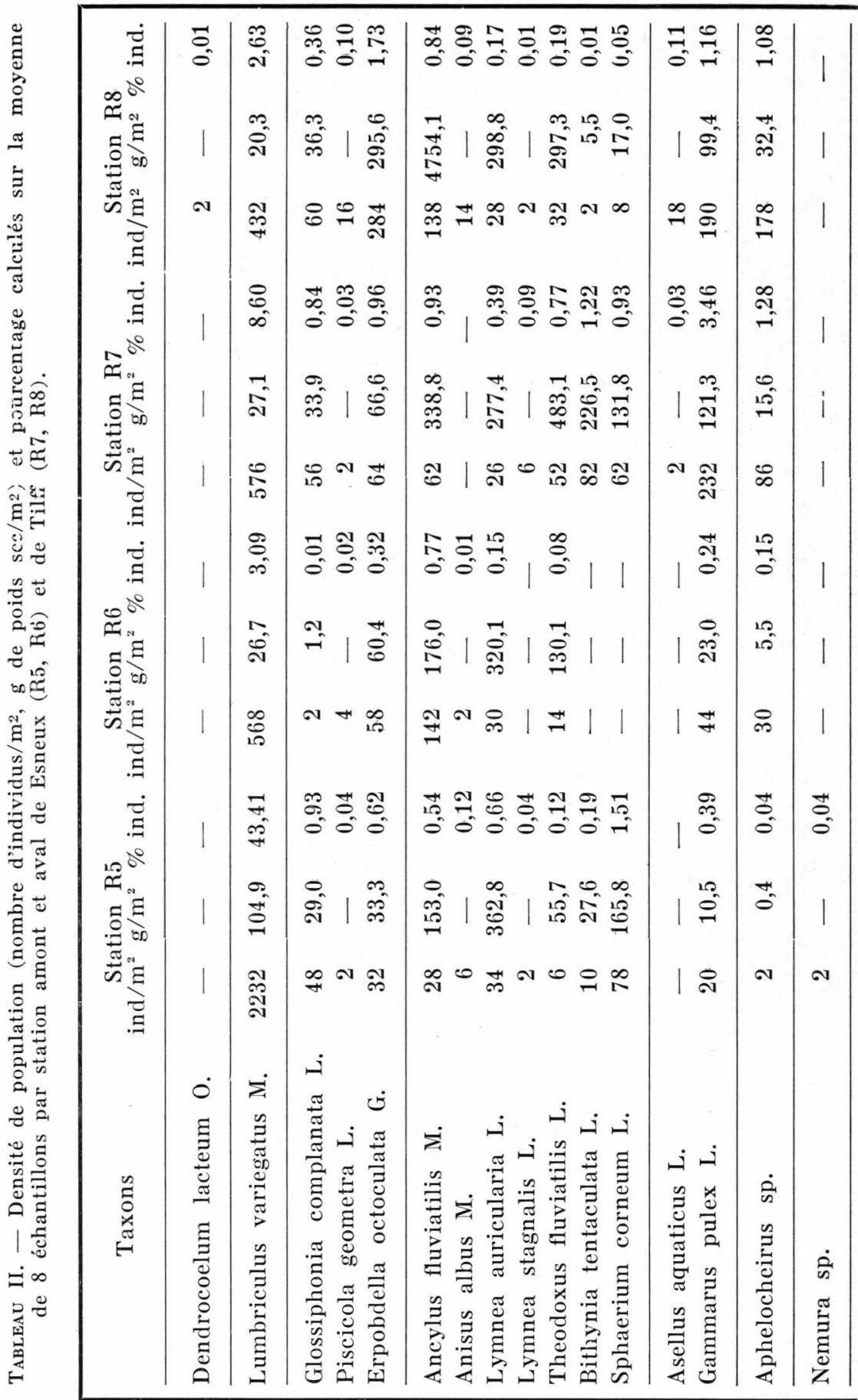




\begin{tabular}{|c|c|c|c|c|c|}
\hline $\begin{array}{l}\mathbb{0} \\
0 \\
0\end{array}$ & 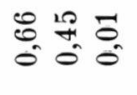 & 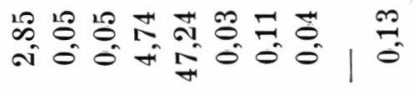 & 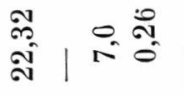 & & 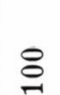 \\
\hline$\frac{-10}{10}$ & ले बे। & 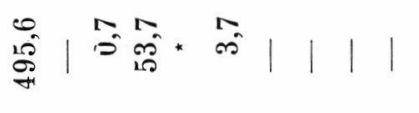 & 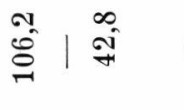 & & 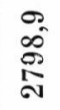 \\
\hline 음 & 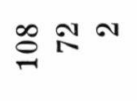 & 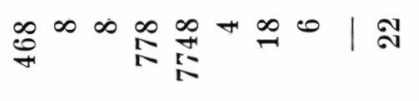 & 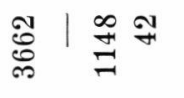 & & 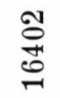 \\
\hline $\begin{array}{l}8 \\
0 \\
0 \\
0\end{array}$ & $\stackrel{\infty}{\infty} \stackrel{\infty}{\infty} \overbrace{0}^{20}$ & 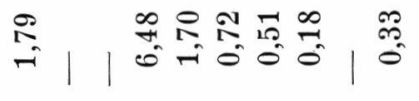 & 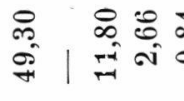 & & ○ \\
\hline in & on & 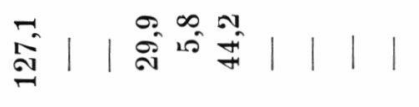 & $\stackrel{\infty}{\infty} \mid \stackrel{\infty}{\infty}$ & & مิ \\
\hline 압 تే & 冓 & 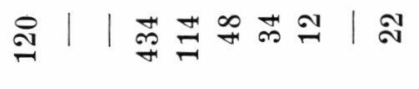 & 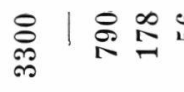 & & ஆ \\
\hline $\min _{0}^{\infty}$ & 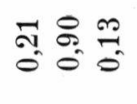 & 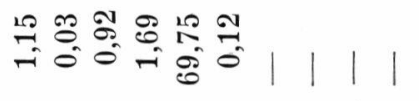 & 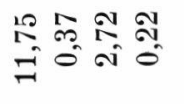 & & $\stackrel{8}{\circ}$ \\
\hline$=\frac{0}{\infty}$ & $\begin{array}{ll}20 \\
-1\end{array}$ & 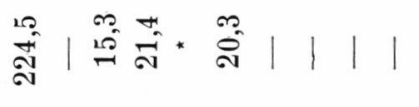 & $\begin{array}{l}10, \infty \\
\text { ป̂ं } \\
0\end{array}$ & & ָூ \\
\hline పా & $\stackrel{\infty}{\infty} \triangleq$ & 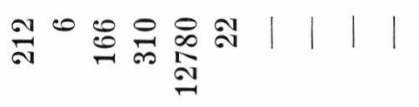 & 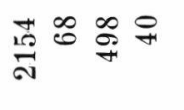 & & $\begin{array}{l}\infty \\
\infty \\
\infty \\
\infty\end{array}$ \\
\hline$\stackrel{0}{2} \underset{-1}{=}$ & 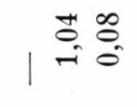 & 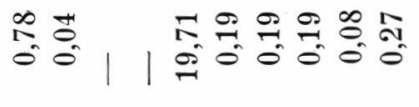 & 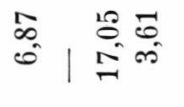 & & $\stackrel{8}{\ominus}$ \\
\hline$\vec{\pi}$ & $|\overrightarrow{\text { जे }}|$ & 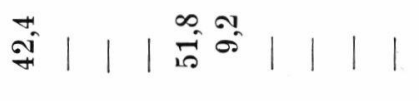 & $\stackrel{\infty}{\stackrel{\infty}{=}} \mid \stackrel{\infty}{\infty}$ & & $\stackrel{\Xi}{\Xi}$ \\
\hline$\circ 8$ & | & 윰 & $\underset{\sim}{\mathbb{1}} \mid \underset{\infty}{\infty} \underset{\infty}{\infty}$ & & $\frac{10}{10}$ \\
\hline 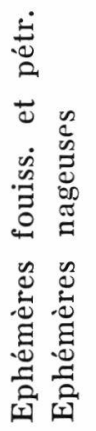 & 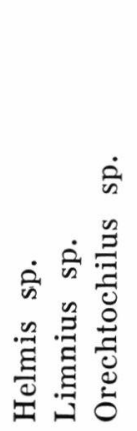 & 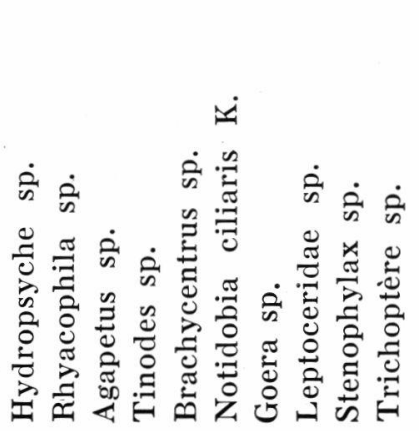 & 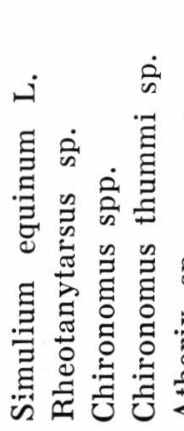 & 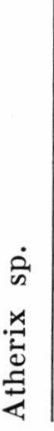 & 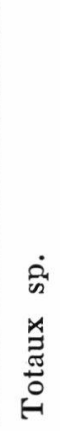 \\
\hline
\end{tabular}


seuses et pétricoles, des Ephémères nageuses et du Trichoptère Hydropsyche sp.

La structure biocénotique est assez bouleversée entre les deux stations amont et aval d'Esneux, mais cela est à mettre en relation avec le recrutement de certains taxons dont Brachycentrus $s p$. est le plus bel exemple.

4. - Tilff (tableau II) présente également une densité en lnvertébrés beaucoup plus grande à sa station aval $\mathbf{R}_{8}$. L'importance de cette augmentation provient surtout du recrutement en Brachycentrus sp. après l'éclosion des œufs. Néanmoins, abstraction faite de ce taxon, la densité de population de nombreux taxons s'accroît en aval de Tilff, ce sont : Erpobdella octoculata, Ancylus fluviatilis, Hydropsyche sp., etc...

La biomasse totale présente également une plus grande importance à la station aval de Tilff. Son augmentation se manifeste surtout chez les taxons suivants : Erpobdella octoculata, Ancylus fluviatilis, Ephémères fouisseuses et pétricoles, Ephémères nageuses, Hydropsyche sp.

Enfin, la structure biocénotique des deux stations est dans l'ensemble fort semblable, exception faite pour Brachycentrus sp.

En conclusion, le phénomène observé en aval de Hamoir, de Comblain-au-Pont et d'Esneux se manifeste encore à Tilf. Les structures biocénotiques restent dans !'ensemble fort semblables, si ce n'est quelques différences dues au recrutement (éclosion) chez certains taxons. Par contre, nous constatons, en aval de ces quatre villes, une augmentation nette de la densité de population et de la biomasse de certains taxons : l'Achète : Erpobdella octoculata, les Mollusques : Ancylus fluviatilis et Lymnea auricularia, le Crustacé Gammarus pulex, les Ephémères, les Trichoptères et les Diptères.

En conséquence, l'examen global des populations d'Invertébrés met en évidence un enrichissement des stations aval en rapport avec l'apport d'eaux résiduaires riches en matières organiques.

\section{- Stations de barrage.}

Le tableau III rassemble les données recueillies aux trois stations de barrage étudiées : $B_{1}$, la Gombe, $B_{2}$, Esneux, $B_{3}$, Colonster.

1. - La première station de la Gombe présente une densité d'Invertébrés assez faible dont la biomasse est assez négligeable. La structure biocénotique montre l'importance des populations de Lumbriculus variegatus et de Chironomus du groupe thumni. Chaque autre taxon représente moins de $20 \%$ de la population en macroinvertébrés. 


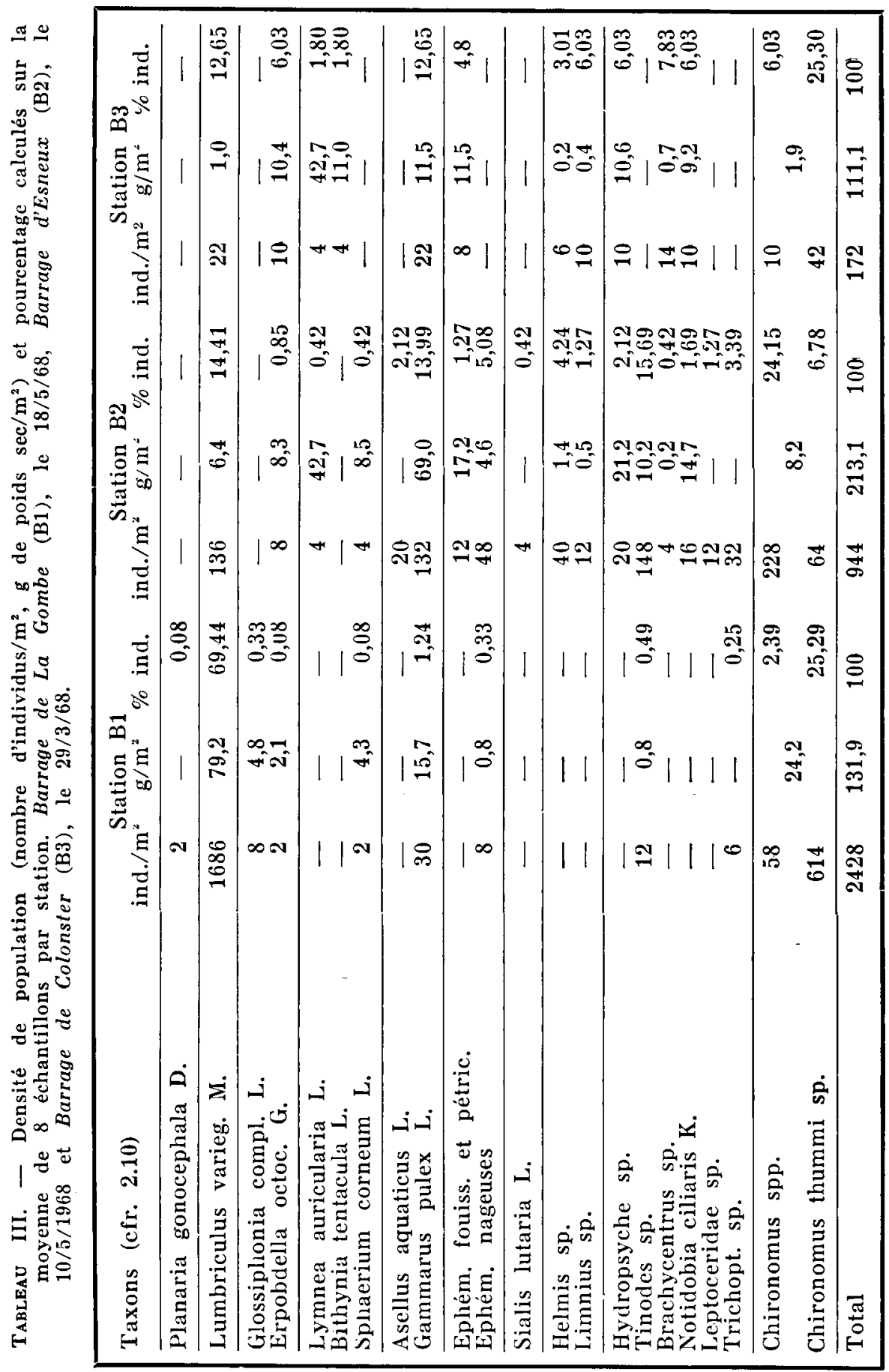


2. - La station de barrage d'Esneux $B_{2}$ présente une densité de population totale très faible, de biomasse également négligeable. La structure biocénotique, encore diversifiée, montre l'importance du Diptère : Chironomus sp., du Trichoptère : Tinodes sp., du Crustacé : Gammarus pulex et de l'Oligochète Lumbriculus variegatus.

3. - La dernière station de barrage à Colonster $\left(\mathrm{B}_{3}\right)$ est encore beaucoup plus pauvre que la précédente. La structure biocénotique reste complexe, aucun taxon n'est vraiment dominant si ce n'est le groupe thummi.

Les stations de barrage présentent une densité de population beaucoup plus faible et une biomasse très réduite par rapport aux stations d'eau courante. Et pourtant rien ne prouve un effet nocif des eaux résiduaires car immédiatement en aval du barrage, la diversité des espèces et la densité de population augmentent fortement. C'est pourquoi il nous semble logique de mettre cette réduction de la biomasse en rapport avec 'ia hauteur d'eau passant de $0,50 \mathrm{~m}$ (rapides) à $1,50 \mathrm{~m}$ (stations de barrage) et réduisant pour le fond de la rivière l'effet photosynthétique de la lumière. En conséquence, la production primaire devient très faible et les organismes en dépendant ne trouvent plus les conditions favorables à leur développement. Un fait d'observation corrobore cette hypothèse : l'abondance du recouvrement d'algues sur le substrat des stations d'eau courante et l'absence presque totale de ce recouvrement au niveau du substrat des stations de barrage.

\section{2. - Type de distribution de quelques espèces.}

La connaissance du mode de répartition des individus d'une espèce, de l'importance et de la permanence des groupes qui peuvent se constituer, est nécessaire pour bien comprendre le comportement d'une population et en apprécier correctement la densité par échantillonnage [VIBERT et LAGLER, 1961]. L'écologiste se doit donc de connaitre la répartition réelle des individus dans les habitats avant de procéder à toute analyse statistique. C'est pourquoi nous avons cherché à quel type des trois grands modes de répartition appartiennent les espèces les plus importantes et les mieux représentées dans les habitats d'eau courante.

Rappelons que la distribution est «non agrégative 》 lorsque la variance est inférieure ou égale à la moyenne. Par contre, si la variance est supérieure à la moyenne, la distribution est dite agrégative, c'est-à-dire que les individus se répartissent par groupes au hasard [NEF, 1967].

Le tableau IV rapporte la moyenne et la variance observées à chaque station d'eau courante $(R)$. Comme nous avons effectué 


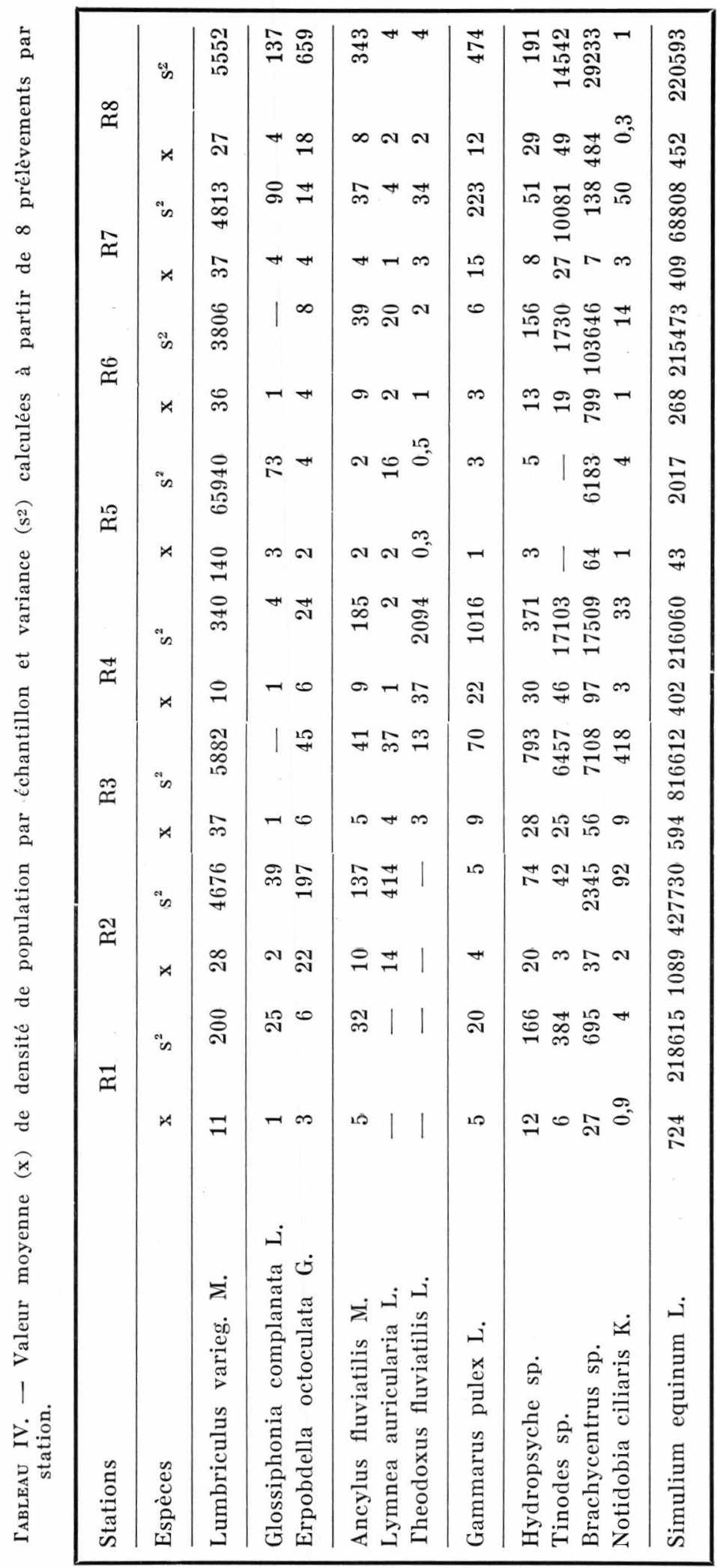


8 prélèvements par station, la moyenne et la variance ont été calculées à partir de ces 8 échantillons. Nous nous sommes limités cependant à l'étude de 12 espèces que nous considérons comme les plus représentatives des rapides et les plus importantes au point de vue biomasse. Ces espèces appartiennent à 4 grandes classes zoologiques : Annélides dont 1 Oligochète et 2 Achètes, Mollusques, Crustacés et Insectes.

L'Oligochète Lumbriculus variegatus présente à chaque station une variance $\left(s^{\prime}\right)$ nettement supérieure à la moyenne $(x)$. Les 2 Achètes : Glossiphonia complanata et Erpobdella octoculata ont également à chaque station une variance $\left(s^{2}\right)$ supérieure à la moyenne $(x)$. Les trois Mollusques, le Crustacé : Gammarus pulex, les 4 Trichoptères et le Diptère Simulium equinum présentent également à chaque station une variance $\left(s^{2}\right)$ nettement supérieure à la moyenne $(x)$. Nous pouvons donc dire que ces 12 espèces appartenant à 4 grandes classes zoologiques ont une distribution agrégative. Autrement dit, les individus de chaque espèce étudiée sont distribués par groupes au hasard.

\section{3. - Analyse de variance.}

Les différences de densité des populations observées en amont et en aval des 4 villes déversant leurs affluents dans l'Ourthe liégeoise supérieure sont soumises à l'analyse de variance. Afin d'homogénéiser les résultats, nous avons opéré la transformation logarithmique $(\log x+1)$ des données originales (nombre d'individus par échantillon). La réalisation de l'analyse qui porte sur les échantillons de plusieurs observations est effectuée selon les notes de cours de Dagneire [1966] et les consejls de L. Nef du Centrum voor Bosbiologisch Onderzoek à Genk. En fait, il s'agit de tester les différences de densité observées pour quelques espèces en fonction de 3 facteurs : situation $S$ (amont ou aval des localités susceptibles de polluer la rivière), milieu $M$ (présence ou absence d'herbier à renoncules) et enfin localité $L$. A cette fin, 3 rapports de variance sont effectués : le premier, situation/interaction $S$ et $L$, le second : milieu/interaction $M$ et $L$, et le troisième : interaction entre $\mathbf{S}$ et $\mathbf{M}$ /interaction entre $\mathbf{S}$. M. et $\mathrm{L}$. La comparaison de ces variances se fait avec respectivement 1 et 3 degrés de liberté [colonne F (S.M.L.)]. Un second type de test $F$ est effectué en groupant les différentes interactions entre elles [colonne $\mathbf{F}(\mathbf{S}+\mathbf{M}) \mathrm{L}]$ de façon à augmenter le nombre de degrés de liberté de la variance servant de base de comparaison. Le nombre de degrés de liberté devient respectivement 1 et 9 et grâce à cela certains $F$ peuvent devenir significatifs.

Signalons encore que tous les calculs ont été effectués sur des 
données transformées en logarithmes à 7 décimales. Enfin les différences de densité de populations de 11 espèces ont été testées grâce à J.-M. Raboux qui a mis au point le programme Fortran pour l'ordinateur IBM 7040 du CECTI (Centre de Calcul et de Traitement de I'Information de l'Institut de Mathématiques à Liège).

1. - Pour l'espèce Lumbriculus variegatus, l'analyse de variance entre les trois facteurs situation, milieı et localité révèle l'absence de toute différence statistiquement significative. L'étude statistique n'accorde donc aucun crédit aux différences de densité de population envisagées aussi bien sous l'angle situation (amont, aval), milieu (présence ou absence d'herbiers) ou localité (proximité de l'embouchure). Il faut conclure que ces différences ne sont que l'illustration de la répartition agrégative des individus.

2. - Pour la population de l'Achète Glossiphonia complanata, l'analyse de variance, effectuée par le test $F$ (S.M.L.), de même que par le test $F(S+M)$ L, révèle que les différences observées en fonction des trois facteurs ne sont jamais significalives. L'étude statistique démontre done l'absence de différences significatives dans la densité de population de Glossiphonia complanata en rapport avec la situation amont ou aval, la présence ou l'absence d'herbiers à Renoncules et les localités.

3. - La population de l'Achète Erpobdella octoculata (tableau V) présente au total un nombre d'individus plus élevé pour la situation aval. Les différences observées en fonction du milieu semblent sans grande importance de même que celles observées en fonction des localités.

L'analyse de variance par le test F (S.M. et L.) comparant les variances des interactions séparées, montre l'absence de toute différence significative dans les densités de Erpobdella en fonction des trois facteurs envisagés. Par contre, le test $F(S+M) L$ révèle l'existence de différences significatives en fonction du facteur situation. L'analyse de variance révèle donc que l'accroissement de densité de la population de Erpobdella octoculata observé en aval de chaque localité est statistiquement significatif. L'interprétation biologique logique est de mettre en relation cet accroissement de densité avec l'apport d'effluents dont les eaux résiduaires non toxiques enrichissent la rivière.

4. - Pour le Mollusque Ancylus fluviatilis, le test F de Fisher qui compare les variances des deux facteurs étudiés aux variances des interactions séparées : F (S.M.L.) ne doune aucun résultat significatif. Le groupement des interactions $F(S+M) L$ ne révèle pas non plus de différence significative. $\mathrm{Du}$ point de vue biologique, la situation amont ou aval, la présence ou l'absence 
TableaU V. - Etude de la population de Erpobdella octoculata

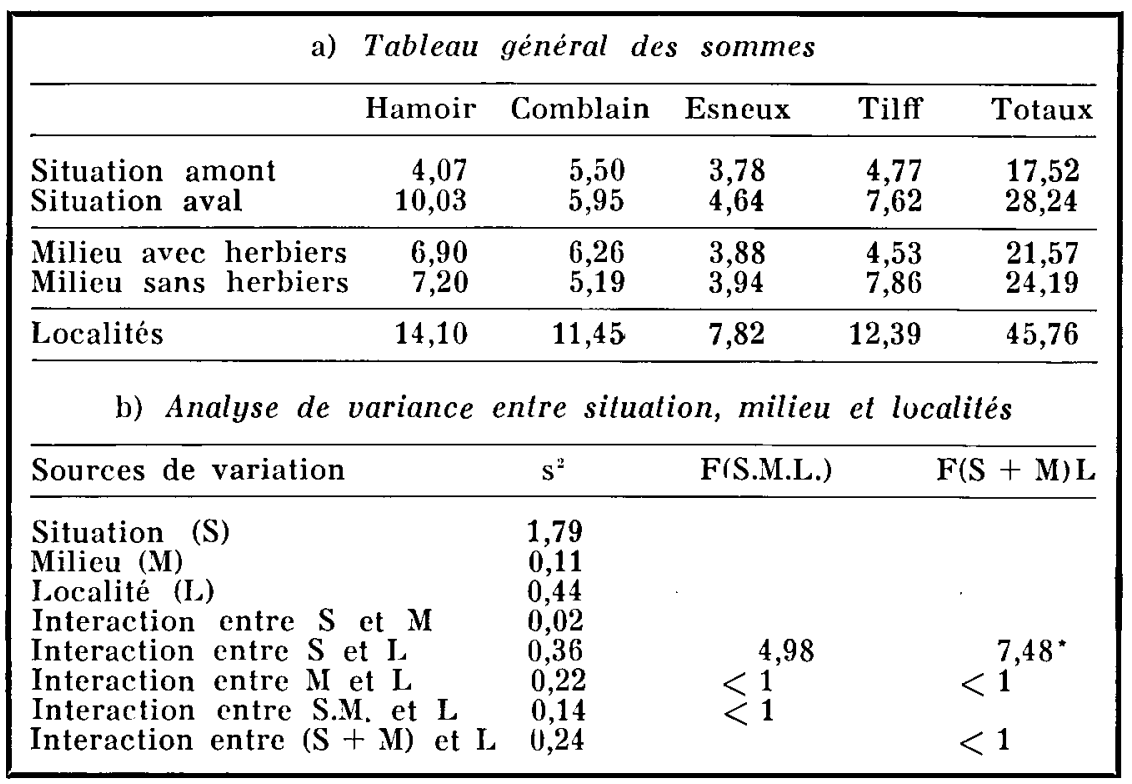

d'herbiers, la localisation des villes sur le parcours de l'Ourthe ne peuvent expliquer les différences de densité de population observées pour l'espèce Ancylus fluviatilis. Ceci nous amène à nuancer les conclusions basées sur l'augmentation de la biomasse en Ancylus fluviatilis aux stations aval des quatre localités étudiées. D’après l'analyse statistique, cet accroissement n'est pas en rapport avec la situation aval mais résulterait plutôt du hasard.

5. - Le second Mollusque étudié : Lymnea auricularia (tableau VI) présente au total une densité de population plus importante pour la situation aval. Toutefois, celte différence est positive ou négative selon les localités. Quant au milieu, l'examen des totaux montre nettement une préférence pour les herbiers. Cette différence se retrouve positive à chaque localité.

L'analyse de variance révèle par les deux types de test $F$ que seules les différences observées en fonction du milieu sont significatives. En termes biologiques, l'accroissement de densité en Lymnea auricularia au niveau des herbiers à Renoncules traduit un préférendum écologique net. Par contre, il n'y a aucune relation statistiquement valable entre la situation et l'augmentation de densité de cette population.

6. - L'analyse de variance pour le dernier Mollusque étudié : Theodoxus fluviatilis ne révèle, pour aucun des trois facteurs envisagés, de différences significatives. Autrement dit, les diffé- 
Tablead VI. - Etude de la population de Lymnea auricularia

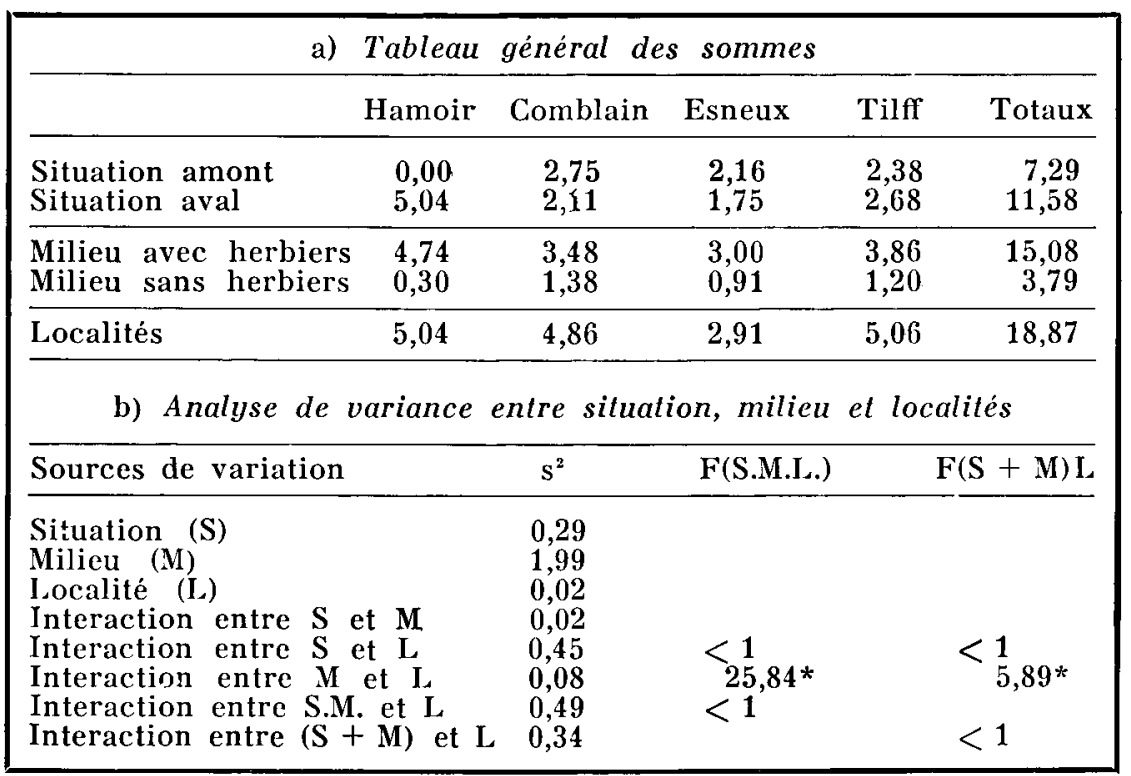

rences de densité observées chez Theodoxus fluviatilis semblent simplement dues au hasard.

7. - L'analyse de variance pour le seul Crustacé étudié : Gammarus pulex, ne décèle aucune différence significative en fonction des trois facteurs étudiés : situation, milieu et localités. Autrement dit, les différences de densité de population notamment observées en fonction du milieu ne sont pas statistiquement explicables et résultent plutôt du hasard.

8. - La population du Trichoptère Hydropsyche sp. (tableau VII) présente au total un nombre plus important d'individus en aval qu'en amont et cette différence se retrouve plus ou moins égale dans chaque localité. Nous constatons également au total qu'il $\mathbf{y}$ a plus d'individus en l'absence d'herbiers. Toutefois, cette différence ne se retrouve pas égale aux différentes localités : les stations de Hamoir, Comblain et Esneux présentent une augmentation de densité de population en l'absence d'herbiers; par contre, aux stations de Tilff, nous constatons une diminution du nombre d'individus en l'absence d'herbiers. Enfin, les différences observées en fonction des localités sont importantes.

L'analyse de variance révèle que les différences observées en fonction de la situation sont significatives par le test F (S.M.L.). Le même résultat est obtenu par le test $F(S+M)$. Les autres 
Tableau VII. - Etude de la population de Hydropsyche sp.

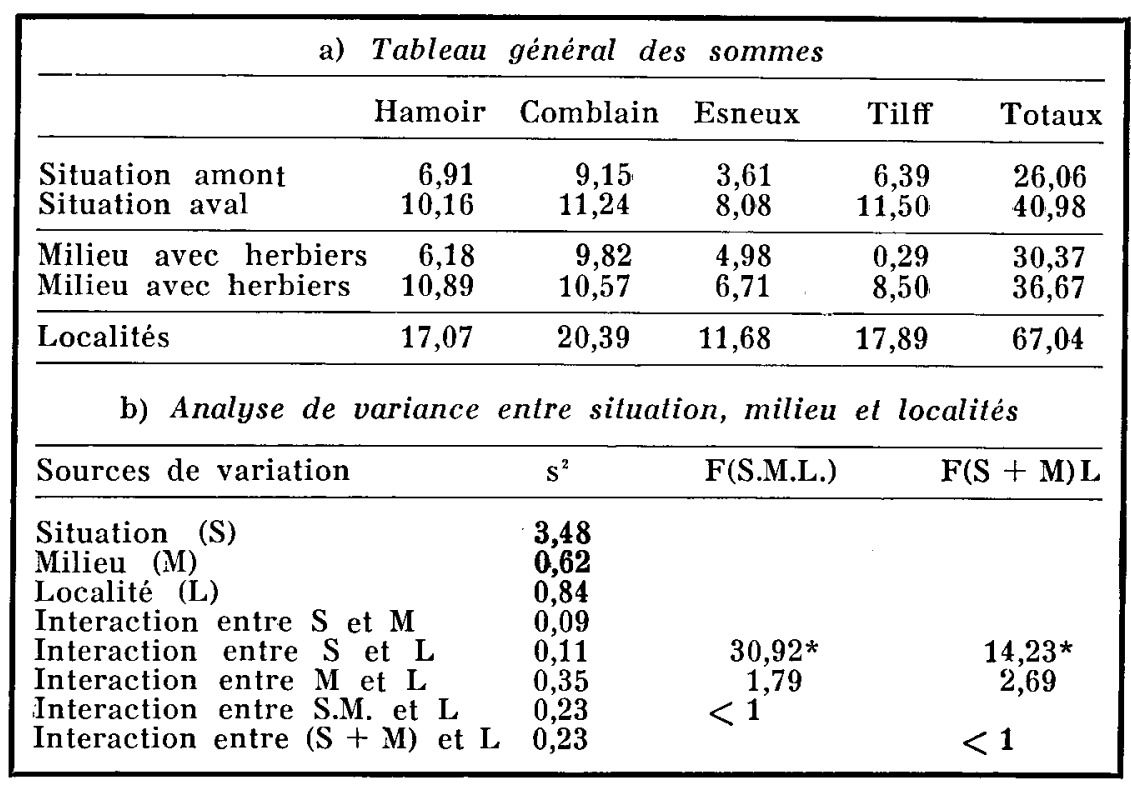

facteurs testés : milieu et localités, n'interviennent pas dans la répartition des individus.

Du point de vue biologique, l'augmentation du nombre d'individus d'Hydropsyche $s p$. déjà décelée par l'examen global des tableaux I et II est en rapport direct avec la situation aval. En d'autres termes, le déversement d'effluents dans la rivière est en relation avec l'augmentation de la densité de population d'Hydropsyche $s p$. Ce qui veut dire que les eaux résiduaires déversées dans l'Ourthe permettent l'accroissement de lá biomasse en Hydropsyche $s p$.

9. - Le tableau VIII a trait au Trichoptère Tinodes $s p$. D'une façon générale, il y a plus d'individus en aval qu'en amont mais les différences ne se retrouvent pas positives pour les quatre localités étudiées. Le milieu sans herbiers présente une densité de population plus élevée en aval et les différences se retrouvent égales aux différentes localités.

L'analyse de variance révèle que les différences observées en fonction du facteur milieu sont significatives par le test F (S.M.L.). Il n'y a pas de différences significatives en fonction des autres facteurs. Le déversement d'effluents dans la rivière n'a aucun effet statistiquement décelable sur la population de Tinodes sp. L'analyse de variance révèle toutefois un préférendum écologique marqué pour un substrat caillouteux, graveleux, dépourvu d'herbiers. 
Tableau VIII. - Etude de la population de Tinodes sp.

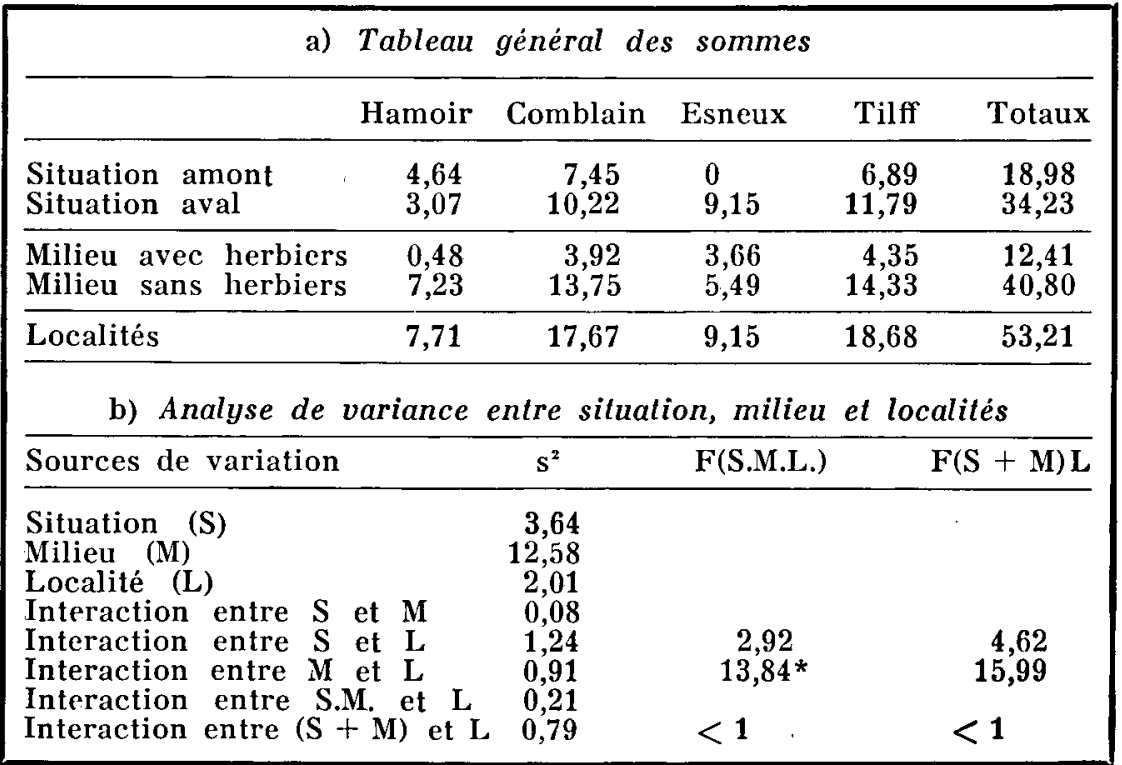

10. - L'analyse de variance pour le dernier Trichoptère étudié : Notidobia ciliaris révèle l'absence de toute différence significative. Les trois facteurs étudiés : situation, milieu et localités n'ont donc aucun effet sur la répartition des organismes.

11. - Enfin le Diptère : Simulium equinum (tableau IX) présente au niveau de chaque localité des densités de population plus grandes pour la situation aval. La présence d'herbiers est nettement favorable.

L'analyse de variance par les deux types de test $F$ révèle que seules les différences de densité de population observées en fonction du facteur milieu sont hautement significatives. Du point de vue écologique, il faut donc considérer que les différences de densité de population observées en fonction de la situation aval et des localités sont dues au hasard. Par contre, l'analyse statistique met en évidence le préférendum écologique de Simulium equinum pour les herbiers à Renoncules.

\section{4. - CONCLUSIONS ET RESUME}

1. - L'étude biologique quantitative effectuée aux stations d'eau courante $(R)$ révèle : 
TAbleau IX. - Etude de la population de Simulium equinum

\begin{tabular}{|c|c|c|c|c|c|}
\hline \multicolumn{6}{|c|}{ a) Tableau général des sommes } \\
\hline & Hamoir & Comblain & Esneux & Tilff & Totaux \\
\hline Situation amont & 11,16 & 7,73 & 6,18 & \multirow{2}{*}{$\begin{array}{l}11,89 \\
12,57\end{array}$} & 37,16 \\
\hline Situation aval & 13,77 & 8,01 & 7,60 & & 41,95 \\
\hline Milieu avec herbiers & 23,03 & 14,27 & 11,87 & \multirow{2}{*}{$\begin{array}{r}20,04 \\
4,42\end{array}$} & 69,21 \\
\hline Milieu sans herbiers & 1,90 & 1,47 & 2,11 & & 9,90 \\
\hline Localités & 24,93 & 15,74 & 13,98 & 24,46 & 79,11 \\
\hline \multicolumn{6}{|c|}{ b) Analyse de variance entre situation, milieu et localités } \\
\hline \multicolumn{2}{|l|}{ Sources de variation } & $\mathrm{s}^{2}$ & \multicolumn{2}{|l|}{ F(S.M.L.) } & $\mathbf{F}(\mathbf{S}+\mathbf{M}) \mathbf{L}$ \\
\hline & 0,36 & \multicolumn{2}{|l|}{ ' } & \multirow[b]{2}{*}{$\because$} \\
\hline & & 54,97 & . & & \\
\hline & 2,05 & - & & \\
\hline Interaction entre $\mathrm{S}$ et & et $\mathrm{M}$ & 0,16 & & \multirow{4}{*}{\multicolumn{2}{|c|}{ 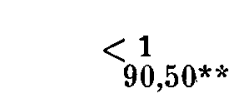 }} \\
\hline \multicolumn{2}{|c|}{ Interaction entre $\mathrm{S}$ et $\mathrm{L}$} & 0,06 & \multirow{3}{*}{$\begin{array}{c}5,57 \\
37,65 * *\end{array}$} & & \\
\hline \multirow{2}{*}{\multicolumn{3}{|c|}{$\begin{array}{l}\text { Interaction entre } \mathrm{M} \text { et } \mathrm{L} \\
\text { Interaction entre } \mathrm{S} . \mathrm{M} \text {. et } \mathrm{L} \\
\mathbf{1 , 4 6} \\
0,30\end{array}$}} & & & \\
\hline & & & & & \\
\hline Interacticn entre $(\mathrm{S}+$ & $+\mathrm{M})$ et $\mathrm{L}$ & - 0,61 & $<1$ & \multicolumn{2}{|r|}{$<1$} \\
\hline
\end{tabular}

a) une augmentation de la densité de population aux stations aval des quatre localités envisagées (Hamoir amont : $7650 \mathrm{ind} / \mathrm{m}^{2}$ et en aval : $12136 \mathrm{ind} / \mathrm{m}^{2}$, etc...);

b) un accroissement systématique de la biomasse en aval de ces quatre localités (Hamoir amont : $111 \mathrm{~kg} / \mathrm{Ha}$ et aval : $228 \mathrm{~kg} / \mathrm{Ha}$, etc...);

c) des structures biocénotiques comparables deux à deux entre stations amont et aval où les populations les plus denses appartiennent aux mêmes espèces : à Hamoir : Simulium equinum (76 et $72 \%)$, Brachycentrus sp. (6 et $5 \%$ ), Chironomus spp. (4 et $4 \%)$, Lumbriculus variegatus (2 et $4 \%$ ), Hydropsyche sp. (3 et $3 \%)$, Tinodes sp. (2 et $1 \%)$, etc...

Le déversement des effluents des quatre localités de Hamoir, Comblain-au-Pont, Esneux et Tilff enrichit les eaux de la rivière en matières organiques (DBO augmente) et a pour conséquence d'augmenter en aval la biomasse secondaire. Du point de vue piscicole, les stations aval présentent donc une plus grande quantité de nourriture disponible.

2. - L'analyse biologique quantitative effectuée aux stations de barrage de la Gombe, d'Esneux et Colonster, décèle par rapport aux stations d'eau courante : 
a) une diminution considérable des densités de population des macro-invertébrés (La Gombe : 2428 ind $/ \mathrm{m}^{2}$ ), (Esneux : 944 ind $\left./ \mathrm{m}^{2}\right)$ et (Colonster : 172 ind $\left./ \mathrm{m}^{2}\right)$;

b) une chute importante de la biomasse (La Gombe : $14 \mathrm{~kg} / \mathrm{Ha}$, Esneux : $21 \mathrm{~kg} / \mathrm{Ha}$, Colonster : $11 \mathrm{~kg} / \mathrm{Ha}$ );

d) une biocénose moins diversifiée mais encore complexe à Esneux et Colonster.

Les stations de barrage sont beaucoup plus pauvres que les stations d'eau courante et la quantité de nourriture pour le poisson $\mathrm{y}$ est très faible. Cet abaissement considérable de la biomasse secondaire est à mettre en relation avec l'augmentation de la hauteur d'eau passant de $0,50 \mathrm{~m}$ aux stations d'eau courante à 1,5-3 m aux stations de barrage, réduisant pour le substrat de la rivière l'effet photosynthétique de la lumière. En effet, lorsque la profondeur d'eau passe de $0,20 \mathrm{~m}$ à $1 \mathrm{~m}$, l'absorption de la lumière est de $50 \%$ [Damseaux, J., 1968]. En conséquence, la production primaire devient très faible et les organismes en dépendant ne trouvent plus de conditions aussi favorables à leur développement.

3. - La vérification du type de distribution des taxons à densité de population élevée dont l'Oligochète Lumbriculus variegatus, les deux Achètes : Erpobdella octoculata et Glossiphonia complanata, le Crustacé Gammarus pulex, quatre Trichoptères : Hydropsyche sp., Tinodes sp., Brachycentrus sp., Notidobia ciliaris et un Diptère : Simulium equinum montre pour tous un type de distribution agrégatif, la variance étant toujours supérieure à la moyenne.

4. - L'analyse statistique effectuée par ordinateur IBM 7040 sur une dizaine d'espèces parmi les plus importantes démontre que :

a) Les différences de densité de population de Lumbriculus variegatus, Glossiphonia complanata, Ancylus fluviatilis, Theodoxus fluviatilis, Gammarus pulex et Notidobia ciliaris n'ont aucune relation avec les trois facteurs envisagés : situation amont ou aval, milieu avec ou sans herbiers, et localisation des villes. Ces différences observées ne reflètent que leur répartition par groupes au hasard.

b) Par contre, l'accroissement de densité de population de Erpobdella octoculata et Hydropsyche sp. est statistiquement en rapport avec la situation aval. En conséquence, le calcul statistique vient confirmer l'impression générale d'enrichissement de 
la rivière par le déversement des effluents urbains de Hamoir, Comblain-au-Pont, Esneux et Tilff.

c) Les différences observées dans les densités de population de Lymnea auricularia, Tinodes sp. et Simulium equinum sont statistiquement en rapport avec le milieu «herbiers à renoncules ». L'analyse statistique met ici en évidence leur préférendum écologique pour les herbiers.

\section{QUANTITATIVE STUDY OF THE BENTHOS FROM THE RIVER OURTHE LIEGEOISE (BELGIUM)}

The biocoenotic structure of macroinvertebrates in the Ourthe river is similar in each rapid station (R) above and below a town, but there are more individuals per square meter and the "standing-crop" is always higher in the stations lying below the towns (above Hamoir: $111 \mathrm{~kg} / \mathrm{Ha}$ and below Hamoir: $228 \mathrm{~kg} / \mathrm{Ha})$. These different values for standing crop in the rapid stations could be due to sewage which contains organic matter.

At every slow and deep station (B), the biocoenosis is always poor and the biomass is very low $\left(B_{2}\right.$ : Esneux: $\left.21 \mathrm{~kg} / \mathrm{Ha}\right)$. The markedly different values for standing crop between rapid stations ( $R$ ) and slow derp stations (B) could be due to the depth (1,5 - $3 \mathrm{~m}$ in $\mathrm{B}$ stations). In the deep stations, the primary production on the substratum is lower than in rapid stations $(R)$; it follows that secondary biomass is lower.

In investigating the pattern of spatial distribution of ten species, the distribution of Lumbriculus variegatus, Erpobdella octoculata, Glossiphonia complanata, Gammarus pulex, Notidobia ciliaris, etc. was seen to be clumped at every station.

The results of the statistical study show that the different population densities of Lumbriculus variegatus, Glossiphonia complanata, Ancylus fluviatilis, Theodoxus fluviatilis, Gammarus pulex and Notidobia ciliaris are not statistically significant.

The populations densities of Erpobdella octoculata and Hydropsyche $s p$. are statistically higher in the below town stations. These different values of density and biomass may be explained primarily by trophic factors, without denying the importance of other influences.

The results of this study provide an illustration of the value of statistical methods in a study of the benthos of a river.

\section{QUANTITATIVE UNTERSUCHUNG DES BENTHOS DER OURTHE, GINES BELGISCHEN FLUSSES NAHE LÜTTICH}

Die Struktur der Makroinvertebratenbiozönosen des Ourthe-Flusses ähneln einander an allen Stationen mit schneller Strömung (R), ob sie unterhalb oder oberhalb von Städten gelegen sind. Die Individuenzahlen pro Quadratmeter sind wie die Biomasse jedoch an den Stationen 
unterhalb von Ortschaften immer größer (Beispiel : oberhalb Hamoir $111 \mathrm{~kg} / \mathrm{ha}$, unterhalb Hamoir $228 \mathrm{~kg} / \mathrm{ha}$ ). Die an Stationen schneller Strömung erhöhten Werte der Biomasse könncn auf die Zufuhr organischer Materie durch Abwässer zurückgeführt werden. An allen Stationen langsamer Strömung (B) und größerer Wassertiefe ist die Biozönose ärmer und die Biomasse weit geringer (Station B 2, Esneux $21 \mathrm{~kg} / \mathrm{ha}$ ). Die großen Unterschiede der Biomasse an Stationen schneller Strömung (R) einerseits und an solchen langsamer Strömung (B) anderseits könnte auf die größere Tiefe an letzteren $(1,5-3 \mathrm{~m})$ zurückzuführen sein.

Hier ist auch die Primärproduktion auf dem Substrat geringer als an Stellen rascher Strömung. Schon daraus folgt, dass auch die Sekundärproduktion geringer sein muss. Die Untersuchung der räumlichen Verbreitung ergab, dass in jeder Station Lumbriculus variegatus, Erpobdella octoculata, Glossiphonia complanata, Gammarus pulex, Notidobia ciliaris usw. gemeinsam vorkommen.

Die Ergebnisse der statistischen Untersuchung haben gezeigt, dass die Verschiedenheiten in der Populationsdichte von Lumbriculus variegatus, Glossiphonia complanata, Ancylus fluviatilis, Theodoxus fluviatilis, Gammarus pulex und Notidobia ciliaris statistisch nicht signifikant sind.

Die Populationsdichten von Herpobdella octoculata und Hydropsyche sp. sind statistisch höher in den Stationen unterhalb der Städte. Diese Unterschiede in der Besiedlungsdichte und Biomasse können primär durch trophische Faktoren erklärt werden, allerdings sind andere Einflüsse nicht auszuschliessen.

Die Ergebnisse vorlegender Untersuchung liefern uns ein Beispeilfür den Wert statistischer Methoden beim Studium des Benthos eines Flusses.

\section{TRAVAOX CITÉS}

Dagnelie (P.). 1965. - A propos de quelques méthodes de comparaisons multiples de moyennes. Biométrie Praximétrie, 6, pp. 115-124.

Dagnelie (P.). 1966. - Cours de statistique mathématique. Edit. Association et Maison des Etudiants, Gembloux, 3 vol., 669 p.

Damseaux (J.). 1968. - Etude du pouvoir auto-épurateur de l'Ourthe entre Tilff et Angleur. Inédit, $18 \mathrm{p}$.

Macan (T. T.). 1958. - Methods of sampling the bottom fauna in stony streams. Assoc. intern. lim. théor. et appl. Commun., ${ }^{\circ}{ }^{\circ}$, pp. 1-21.

Micha (J.-C.). 1969. - Etude qualitative des associations d'invertébrés de l'Ourthe liégeoise. Ann. Soc. roy. zool. Belg., 99, 4, pp. 215-236.

Micha (J.-C.). 1970. - Etude des populations d'invertébrés de l'Ourthe liégeoise. Etude du milieu. Tribune du Cebedeau, 23, pp. 62-75.

NEF (L.). 1967. - Les distributions agrégatives et leur application en écologie animale. Biométrie-Praximétrie, $8, n^{\circ} 3-4$, pp. 160-190. 
Vibert (R.) et Lagler (K. F.). 1961. - Pêches continentales. Biologie et aménagement, Dunod, Paris, $720 \mathrm{p}$.

Welch (S. Paul). 1952. - Limnology - New York - Toronto - London, 538 p.

Laboratoire de l'Aquarium (Prof. J.C. Ruwet), Institut de Zoologie de l'Université, Quai Van Beneden, 22, B - 4000 Liège, Belgique. 